\title{
A Rare Case of Infectious Colitis with Ulcers in the Cecum Caused by Mycobacterium Gordonae
}

\author{
Tsutomu Mizoshita, Satoshi Tanida, Takashi Mizushima, Yoshikazu Hirata, Kenji Murakami, \\ Takaya Shimura, Yoshinori Mori, Hiromi Kataoka, \\ Takeshi Kamiya and Takashi Joh
}

\begin{abstract}
A 69-year-old female complained of persistent abdominal pain, and annular ulcers and ulcer scars were detected endoscopically in the cecum. Pathological findings included caseous granulomas with some Langhans giant cells, and Ziehl-Neelsen staining was negative. Mycobacterium gordonae (M. gordonae) was identified by the DNA-DNA hybridization method and culture (Ogawa medium) of biopsy samples from ulcerous cecal lesions. After 6 months of antibiotic therapy, ulcerous cecal lesions were healed, and no acid-fast bacteria were detected by culture of biopsy samples from scar tissue. We believe this is the first report of M. gordonae infection in the alimentary tract.
\end{abstract}

Key words: Mycobacterium gordonae, double balloon endoscopy, non-tuberculous mycobacteria

(Intern Med 50: 2583-2586, 2011)

(DOI: 10.2169/internalmedicine.50.6238)

\section{Introduction}

The incidence of non-tuberculous mycobacteria (NTM) infection is currently increasing in aged individuals and those immunocompromised by immunosuppressive therapy (1-4). NTM are often observed as the primary causative organisms in patients with pulmonary infection. However, there are few reports of NTM infection in the alimentary tract. In most of these, Mycobacterium avium (M. avium) and Mycobacterium intracellulare (M. intracellulare) were detected.

Mycobacterium gordonae (M. gordonae) is a species of NTM commonly found in tap water and soil, and incidentally in human colon lavage specimens. It is known to have low virulence in healthy individuals. This species often infects the respiratory system, particularly the lungs $(5,6)$. Here we report a rare case of infectious colitis caused by $M$. gordonae affecting the cecum of a non-immunocompromised patient.

\section{Case Report}

A 69-year-old female complained of persistent abdominal pains, and was found to have annular stenosis of the cecum by barium enema in another hospital (Fig. 1). Complete colonoscopy was attempted using a normal colonoscope, but the fiber could not be passed through the ascending colon and cecum because of coalescence from previous appendectomy and uterectomy. Therefore, in November 2009 referral was made to our hospital for total colonic examination by double balloon endoscopy (DBE).

At the time of presentation, C-reactive protein (CRP) and erythrocyte sedimentation rate (ESR) were $0.37 \mathrm{mg} / \mathrm{dL}$ (normal range $\leq 0.30 \mathrm{mg} / \mathrm{dL}$ ) and $18 \mathrm{~mm} / \mathrm{h}$ (normal range $\leq 16$ $\mathrm{mm} / \mathrm{h}$ ), respectively. Chest X-ray was normal, and QuantiFERON TB-2 G (Cellestis Ltd., Sydney, Australia) was negative. DBE was performed in December 2009. Ulcers and ulcer scars were arrayed annularly along the haustral fold, and clots and edematous changes were detected in the cecum (Fig. 2A). Pathological findings included areas of marked acute and chronic inflammation, and caseous granulomas 
(Fig. 3A, 3B) with some Langhans giant cells (Fig. 3C, 3D). Ziehl-Neelsen staining was negative. The possibility of $M y$ cobacterium tuberculosis (M. tuberculosis) infection of the cecum was considered, and an antibiotic regimen of isoniazid (INH) $300 \mathrm{mg} /$ day, rifampicin (RFP) $450 \mathrm{mg} /$ day, ethambutol (EB) $750 \mathrm{mg} / \mathrm{day}$, and pyrazinamide (PZA) 1.0 g/day was begun in January 2010. In February 2010, M. gordonae was identified in biopsy samples from ulcerous cecal lesions by the DNA-DNA hybridization method, and by culture (Ogawa medium). INH and PZA administration was discontinued, and from March to June 2010 the patient was given a three-drug regimen: RFP $450 \mathrm{mg} /$ day, EB 750 $\mathrm{mg} /$ day, and clarithromycin (CAM) $800 \mathrm{mg} /$ day.

After 6 months of antibiotic therapy, DBE was again performed. The ulcerous lesions in the cecum were healed, and only annular ulcer scars were detected (Fig. 2B). Pathological investigations revealed an absence of caseous granulomas and improvement of inflammation. No acid-fast bacteria were detected in cultures of biopsy samples from the ulcer scar tissue.

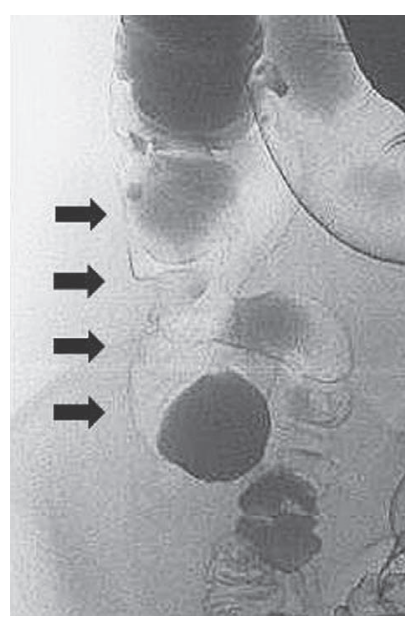

Figure 1. The barium enema showed the stenosis of the cecum (black arrows).
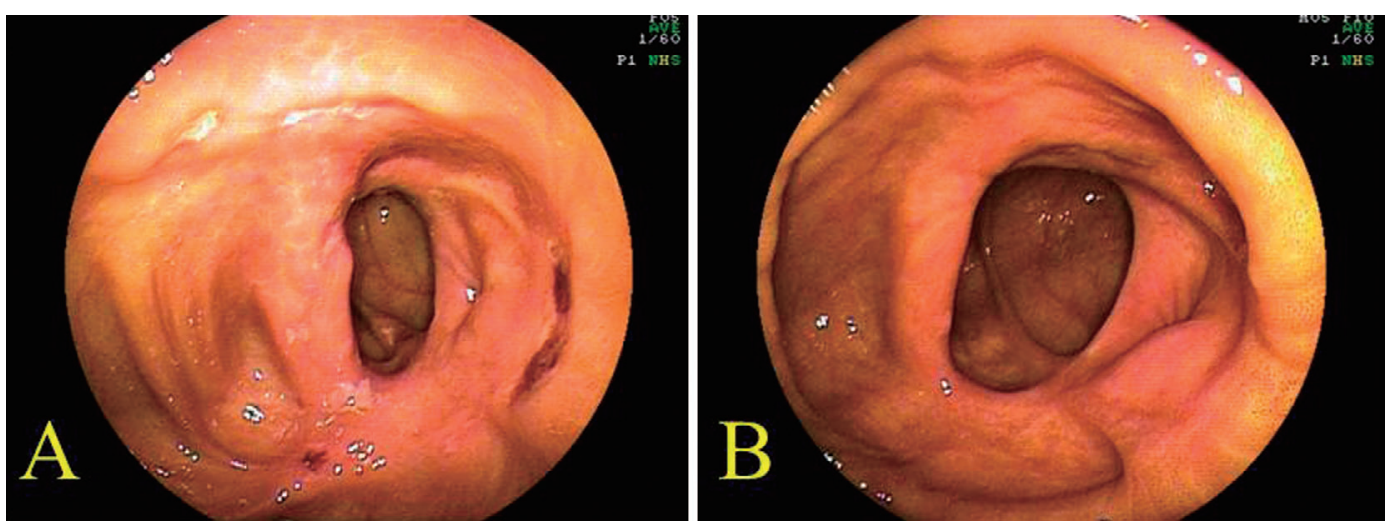

Figure 2. (A) Endoscopy revealed ulcers and ulcer scars in the cecum. (B) After 6 months of antibiotic therapy the ulcers were healed, and only annular ulcer scars were detected.

\section{Discussion}

To the best of our knowledge, this is the first report of infectious colitis with ulceration in the cecum caused by $M$. gordonae infection. Differential diagnosis includes Crohn's disease, which results in deep geographic and serpiginous ulcers in the alimentary tract. Infection with M. tuberculosis was ruled out by the DNA-DNA hybridization method and culture (Ogawa medium) of biopsy samples from ulcerous cecal lesions.

NTM infection in the alimentary tract is associated with the immunodeficient state secondary to human immunodeficiency virus (HIV) infection or use of immunosuppressive agents $(1,2)$. In otherwise healthy individuals, Ueda et al reported an $M$. avium infection in the ileocecum (2), and Fujisawa et al found Mycobacterium nonchromogenicum (M. nonchromogenicum) infection in the cecum and ascending colon (7). Recent reports of NTM colitis are summarized in Table 1. It also has been reported that NTM are isolated from the fluid phase of colonic contents in 17.6-46.3\% of all specimens, suggesting the possibility of mycobacterial contamination during colonoscopies (8). In the present case, $M$. gordonae infection in the cecum was proved by the DNA-DNA hybridization method and culture (Ogawa medium) of biopsy samples from ulcerous cecal lesions.

It has been reported that patients with NTM infection are given antitubercular therapy identical to that used for $M$. $t u$ berculosis infection $(2,7)$. In the present case, first, the antitubercular therapy (INH + RFP + EB + PZA) was begun, but the drug sensitivity test showed resistance to INH, RFP, and EB of $M$. gordonae after 2 months. Therefore, we started the three-drug regimen (RFP + EB + CAM), because the three-drug therapy with RFP + EB + CAM has been used against NTM infection (1, 5, 6). As a result, 2 months of antitubercular drugs (INH + RFP + EB + PZA) and 4 months of antimycobacterial therapy (RFP + EB + CAM) resulted in successfully cured lesions.

It is well-known that the M. tuberculosis infection exhibits the annular ulcers endoscopically in the ileocecum. The 

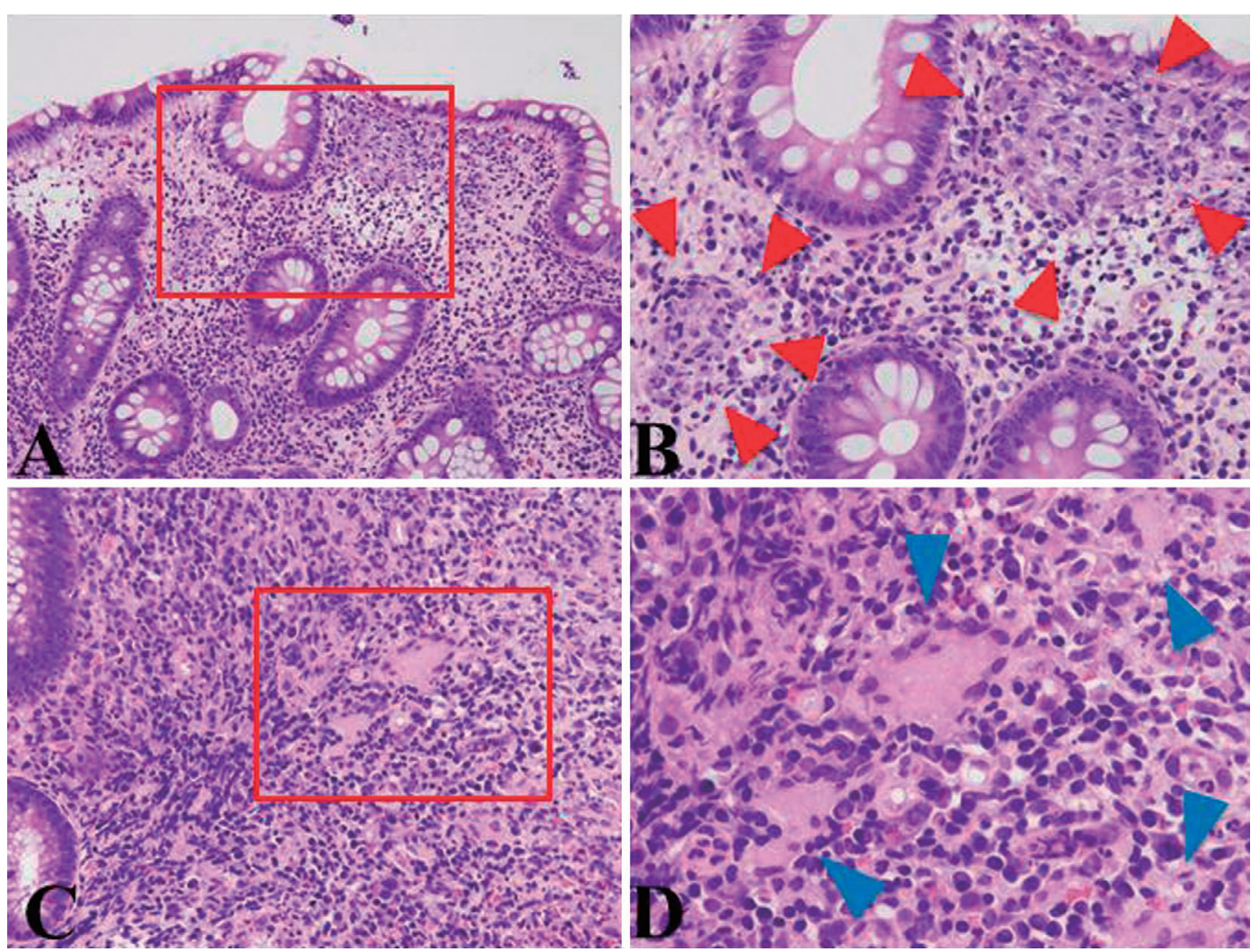

Figure 3. The caseous granulomas (A, red arrowheads in B) and some Langhans giant cells (C, blue arrowheads in $D$ ) were detected pathologically in the biopsy samples from ulcerous cecal lesions. Original magnification, $A, \times 200 ; B, \times 400 ; C, \times 400 ; D, \times 800$.

Table 1. Recent Reports of NTM Colitis

\begin{tabular}{cccccc}
\hline $\begin{array}{c}\text { Reference } \\
\text { number }\end{array}$ & NTM species & Age & Gender & $\begin{array}{c}\text { Immuno- } \\
\text { compromised }\end{array}$ & Therapy \\
\hline 1 & M. avium & 60 & Male & Yes & RFP+EB+CAM \\
2 & M. avium & 40 & Female & No & INH+RFP+EB \\
7 & M. nonchromogenicum & 23 & Female & No & INH+RFP+SM \\
\hline
\end{tabular}

NTM: non-tuberculous mycobacteria; RFP: rifampicin; EB: ethambutol; CAM: clarithromycin; INH: isoniazid; SM: streptomycin

annular ulcers were detected endoscopically in two cases of M. avium infection in the ileocecum $(1,2)$, while Fujisawa et al showed endoscopic findings of ulcers that were irregularly arranged in the $M$. nonchromogenicum infection of the ascending colon (7). In the present case, M. gordonae infection exhibited annular ulcers endoscopically in the cecum.

DBE is useful not only for the observation of the small intestine but also, as in the present patient, in cases where conventional total colonoscopy is difficult (9). In conclusion, we successfully treated an otherwise healthy female having M. gordonae infection of the cecum with 6 months of antibiotic therapy. To the best of our knowledge, this is the first report of M. gordonae infection causing annular ulcers of the alimentary tract.

The authors state that they have no Conflict of Interest (COI).

\section{References}

1. Nguyen HN, Frank D, Handt S, et al. Severe gastrointestinal hemorrhage due to Mycobacterium avium complex in a patient receiving immunosuppressive therapy. Am J Gastroenterol 94: 232-235, 1999.

2. Ueda W, Okawa K, Sano K, et al. Mycobacterium-Avium Enteritis, Report of a case. Stomach and Intestine 44: 1622-1628, 2009 (in Japanese, Abstract in English).

3. Barber TW, Craven DE, Farber HW. Mycobacterium gordonae: a possible opportunistic respiratory tract pathogen in patients with advanced human immunodeficiency virus, type 1 infection. Chest 100: 716-720, 1991.

4. Lessnau KD, Milanese S, Talavera W. Mycobacterium gordonae: a treatable disease in HIV-positive patients. Chest 104: 1779-1785, 1993.

5. Goto R, Katsura H, Yamada K, Motegi T, Kida K. A case of infected bulla caused by Mycobacterium gordonae. Nihon Kokyuki Gakkai Zasshi 40: 26-30, 2002 (in Japanese, Abstract in English). 6. Saeki S, Matsuse H, Shimoda T, Soejima Y, Ohno H, Kohno S. A 
case of pulmonary Mycobacterium gordonae infection with pleural effusion. Nihon Kokyuki Gakkai Zasshi 42: 103-107, 2004 (in Japanese, Abstract in English).

7. Fujisawa K, Watanabe H, Saito H. Primary atypical mycobacteriosis of the intestine: A new clinical entity, report of a case. Stomach and Intestine 25: 95-102, 1990 (in Japanese, Abstract in Eng- lish).

8. Kobayashi Y, Takano T, Hirayama N, Sato N, Shimoide H. Isolation of nontuberculous mycobacteria during colonoscopy. Kekkaku 70: 629-634, 1995 (in Japanese, Abstract in English).

9. Yamamoto H. Double-balloon endoscopy. Clin Gastroenterol Hepatol 3: S27-S29, 2005.

(C) 2011 The Japanese Society of Internal Medicine http://www.naika.or.jp/imindex.html 